\title{
Immediate Effects of Neural Slider and Neural Tensioner on Forward Bending in Subjects with Hamstring Tightness
}

\author{
Jin-yong Lim, PT, Ph.D ${ }^{1}$; Il-woo Lee, PT, BS ${ }^{2}$; Kyoung-don Kim, PT, Ph.D ${ }^{3}$ \\ ${ }^{1}$ Department of Physical Therapy, College of Rehabilitation Sciences, Daegu University, Daegu, Republic of Korea \\ ${ }^{2}$ Department of Physical Therapy, Hansol Hospital, Daegu, Republic of Korea \\ ${ }^{3}$ Department of Physical Therapy, Deagu Health College, Daegu, Republic of Korea
}

Background Two different neurodynamic techniques have been suggested: (i) the slider (ii) the tensioner. However, the effects of slider and tensioner on hamstring flexibility during forward bending have not been directly compared.

Purpose This study aimed to compare the immediate effects of slider and tensioner on forward bending in asymptomatic subjects with hamstring tightness.

Study design Two groups pre- and post-test design

Methods Twenty male subjects with hamstring tightness participated in this study. The subjects were randomly allocated to the slider and tensioner groups, consisting of 10 participants each. To measure hip and lumbar flexion during forward bending, a smart motion sensor was employed. The paired $t$-test was applied to compare pre- and post-intervention within each group. The independent $t$-test was used to compare the hip and lumbar flexion angles between the two groups.

Results Intragroup comparison found that the slider group had a significant increase in hip flexion range of motion at post-intervention. Intergroup comparison found a significant greater change in hip flexion range of motion between pre- and post-intervention in slider group.

Conclusions Based on the results of this study, it was found that sliders are more effective than tensioners in enhancing hamstring flexibility during forward bending in subjects with hamstring tightness.

Key words Flexibility; Forward bending; Hamstring; Slider; Tensioner.

\author{
J Musculoskelet \\ Sci Technol \\ 2021; 5(1): 6-13 \\ Published Online \\ Jun 30,2021 \\ pISSN 2635-8573 \\ eISSN 2635-858
}

Article History

Received 23 Feb 2021

Revised 3 Mar 2021

(1st)

Revised 16 Mar 2021

(2nd)

Accepted 16 Mar 202

\section{CONTACT}

doniee1595@gmail.com Kyoung-don Kim,

Department of Physical

Therapy, Deagu Health

College, Daegu, Republic

of Korea

This is an Open-Access article distributed under the terms of the Creative Commons Attribution Non-Commercial License (http://creativecommons. org/licenses/by-nc/4.0) which permits unrestriced non-copermits unrestricted non-coand reproduction in any meand reproduction in any medium, provided the origina work is properly cited.

\section{INTRODUCTION}

Hamstring is a muscle group that has a tendency to develop tightness, which affects all age groups. ${ }^{1}$ The hamstring muscle affects pelvic motion because it originates from the sciatic surface of the pelvis. ${ }^{2}$ Hamstring flexibility is a critical parameter because a decreased extensibility of this muscle is a factor that predicts injuries, nonspecific back pain, and changes in the lumbopelvic rhythm..$^{3,4}$ Maintaining hamstring flexibility is crucial for restoring musculoskeletal functions, such as the lumbopelvic rhythm. ${ }^{5}$ Carregaro and Coury et al. evaluated decreased hamstring flexibility for the lumbar and pelvic movements during a box lowering task and found that subjects with reduced flexibility showed more lumbar movements and limited pelvic movements during a box lowering task. ${ }^{6}$ Decreased hamstring flexibility limits hip movement while forward bending of the trunk and simultaneously results in excessive lumbar movement. ${ }^{7}$ The peripheral muscles and ligaments behind the lumbar spine are subjected to excessive tension and, consequently, cause low back pain. ${ }^{8}$

The effect of stretching exercises on hamstring flexibility during forward bending is an interesting subject for researchers. Some previous studies have investigated the effects of hamstring stretching on the lumbopelvic rhythm. ${ }^{9,10}$ Hasebe and colleagues performed a finger-floor distance to 
determine the change of hamstring flexibility to lumbopelvic rhythm after dynamic stretching, and reported a significant increase in finger-floor distance after dynamic stretching. ${ }^{9}$ Another study reported that the anterior rotation of the pelvis increased during forward bending after 3 weeks of stretching exercise. ${ }^{10}$

Although abnormal stiffness of muscles and tendons is thought to cause insufficient hamstring flexibility, some authors claim that abnormal mechanosensitivity of the sciatic nerve can cause potentially reduced hamstring extensibility and stretching tolerance. ${ }^{11-13}$ Choi et al. proved that hip flexion decreased during forward bending in patients with tension of the sciatic nerve. ${ }^{12}$

The neurodynamic technique has been used in clinical settings for the mobilization of peripheral nerve and surrounding structures. ${ }^{14}$ The neurodynamic technique has recently been suggested as an alternative to stretching exercises for patients with hamstring tightness by eliminating the nerve factor of tightness without stretching the hamstring muscle. ${ }^{15,16}$ For example, Sharma et al. reported that a significantly greater hamstring flexibility was obtained when the neurodynamic technique was combined with muscle stretching compared to muscle stretching alone. ${ }^{17}$ Butler et al. suggested the use of a slider or tensioner as a means of moving nervous tissues. ${ }^{18}$ The neurodynamic technique can be effective in reducing neural mechanosensitivity and managing hamstring flexibility. ${ }^{19}$ Tensioners are considered to stretch the nervous tissues and increase nervous tension and strain. ${ }^{20}$ For example, Fidel et al. applied tensioner mobilization in the slum position on 27 healthy subjects and reported an increase of about 5.6 degrees in the knee extension angle after tensioner mobilization. ${ }^{21}$ Sliders use a technique that makes nerve structures slide the surrounding adjacent tissues without changing the length of the nerve. ${ }^{17}$ This technique allows the target nerve structure to provide tension in the proximal part through joint movement and relax the tension of the nerve in the distal part, and it performs this sequence in reverse order. ${ }^{22}$ In a study of
Castellote-Caballero et al., the neurodynamic slider was performed for a week, and it was reported that passive straight leg raising (SLR) increased significantly. ${ }^{11}$

Many researchers suggested an increase in hamstring flexibility after applying sliders and tensioners, but they measured them by the knee extension angle and SLR. ${ }^{15,23}$ The effects of slider and tensioner on hamstring tightness during forward bending have not been directly compared. Therefore, this study aimed to compare the immediate effects of neural slider and tensioner on forward bending in subjects with hamstring tightness.

\section{METHODS}

\section{Subjects}

Twenty male subjects $(N=20)$ with hamstring tightness were recruited for this study. All subjects were free of injury at the time of this experiment and had no history of surgery on their lower extremities. They were physically active, but none of them engaged in sports. And they had hamstring muscle tightness, which was explained as achieving less than 60 degrees of knee extension in vertical plane when it was measured in the active knee extension (AKE) test position. ${ }^{24}$ The subjects were randomly assigned to the slider and tensioner groups, consisting of 10 participants each. A coin was tossed to assign subjects to these groups. When the head of the coin came out, the subject was assigned to the slider group; when the back of the coin came out, the subject was assigned to the tensioner group. All the subjects were provided of the aim of this study before they agreed with a consent form. The study was approved by the Ethics and Research Committee of Daegu Health College (\#DHCIRB-2020-09-0006). The general characteristics of the subjects are summarized in Table 1.

\section{Instrumentation}

We used a smart KEMA motion sensor (KOREATECH Co, Ltd., Korea) to measure the range of motion (ROM)

Table 1. General characteristics of subjects

\begin{tabular}{cccc}
\hline Characteristics & Slider group $(N=10)$ & Tensioner group $(N=-10)$ & $p$ \\
\hline Age (years) & $28.90 \pm 3.63$ & $30.40 \pm 4.85$ & 0.44 \\
Weight $(\mathrm{kg})$ & $75.05 \pm 7.85$ & $73.70 \pm 6.60$ & 0.69 \\
Height $(\mathrm{cm})$ & $177.40 \pm 5.69$ & $173.70 \pm 3.05$ & 0.09 \\
Right AKE (degrees) & $25.45 \pm 7.65$ & $25.03 \pm 5.36$ & 0.88 \\
Left AKE (degrees) & $24.53 \pm 7.08$ & $25.07 \pm 4.83$ & 0.84 \\
\hline
\end{tabular}

Data are expressed as mean \pm standard deviation; AKE, active knee extension; ${ }^{*} p<0.05$. 
during knee extension and forward bending. Data were collected at a sampling frequency of $25 \mathrm{~Hz}$ and transmitted to an Android tablet running the Smart KEMA software.

\section{Procedure}

All procedures related to data collection were explained to the subjects. Hamstring tightness of the lower extremity of each subject was measured prior to assignment to the groups. The subjects were screened as patients with hamstring tightness using the AKE test. After hip and lumbar flexion ROMs were measured by a forward bending test, the subjects were divided into each group and received intervention. To examine immediate changes after intervention, the subjects performed forward bending again, and their hip and lumbar flexion ROMs were measured again. At the posttest, we measured immediately without resting period to prevent a decrease of intervention effect. The examiners were double blinded to the pre- and post-test of forward bending measurements.

Hamstring tightness was determined in both legs by the AKE test. The intratester reliability of this test has been reported to be excellent in previous studies. ${ }^{25}$ The subjects performed a supine position on the table with a towel placed between the lumbar vertebrae and the table to minimize the effect of lumbar movement on examination results and help maintain the lumbar curve. In order to maintain 90 degrees flexion of the hip joint, anterior thigh was touched to the fixed bar. The non-test leg was positioned with the hip and knee extension at 0 degrees. The strap was used to place the motion sensor on the lateral malleolus. In order to be familiar with the test, Subjects performed 5 AKEs with their ankle at rest. The subjects performed knee extension actively. The range of the AKE test was recorded for the last 3 seconds. We determined the end point of the AKE test with feelings of discomfort or tightness in the hamstring muscle. Three trials were performed for each leg, and the mean value was calculated for each side. The subjects rested for 1 minute between each test. The range of AKE was recorded as 0 degrees at the start position with 90 degrees knee flexion. Both knees were measured by the AKE test.

Two smart KEMA motion sensors were used to measure changes in the motion of the hip and lumbar spine during forward bending. One sensor was placed on the second spinous process of the sacrum using an adjustable belt, and another was attached to the first spinous process of the lumbar spine using double-sided tape. The motion sensors were calibrated to 0 degrees in a standing position with the subject shoulder-width apart. Then the subject was asked to bend forward for 3 seconds. Subject was instructed to avoid flexion of the knee joints while bending forward. The met- ronome was used to control the bending speed. We determined the tightness or discomfort of the hamstring as the end point of forward bending test (Figure 1). The forward bending test was performed twice, and the mean value was used for data analysis. Hip flexion was obtained from pelvic anterior tilt, and lumbar flexion was calculated from the difference between the two sensors. ${ }^{26}$

\section{Intervention}

For each technique, the subjects were asked to reach the end of available range for each joint motion. At present, the literature on or evidence of the appropriate frequency for active neurodynamic techniques is ambiguous. Therefore, five sets of 30 seconds were applied according to the author's suggestion and clinical experience. ${ }^{27,28}$ However, the application sequence was set randomly. Computer-generated random numbers were used for randomization by an investigator

Slider

The start position of the subject consisted of upper cervical flexion in the supine position, the hip and knee joints

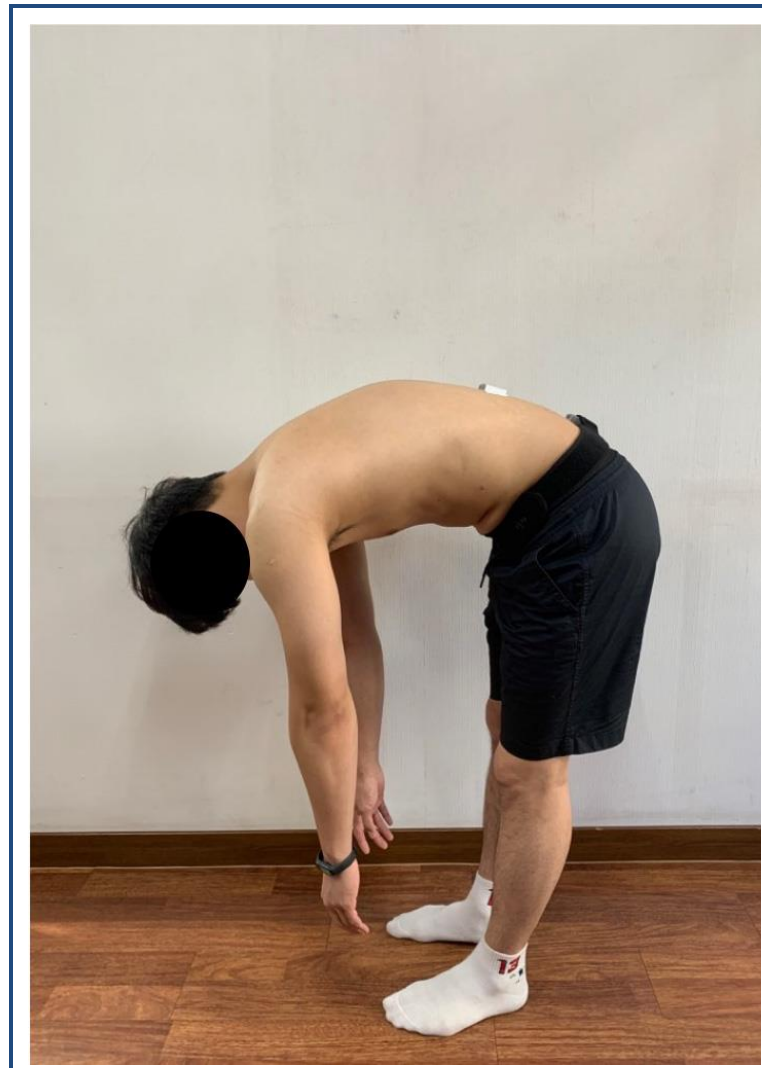

Figure 1. Range of motion measured using the Smart KEMA motion sensor for forward bending of the trunk. 
bent at 90 degrees, and the ankles plantar-flexed. The back of the thigh was wrapped with both hands to fix the thigh bone during the exercise. In this position, the subject performed extension of knee and dorsiflexion of the ankle simultaneously while extending the upper cervical spine. This movement was performed without the feeling of pulling of the nerve tissue in the back of the thigh. Following this, the subject performed upper cervical flexion simultaneously with knee flexion and ankle plantar flexion. The subjects performed five sets of this exercise consecutively with active movement for approximately 30 seconds, taking a rest for 10 seconds in between sets. ${ }^{29}$ After resting for 2 minutes, they performed the same exercise using the opposite lower limb (Figure 2).

\section{Tensioner}

The start position of the subject consisted of upper cervical extension in the supine position, the hip and knee joints bent at 90 degrees, and the ankles plantar-flexed. The back of the thigh was wrapped with both hands to fix the thigh bone during the exercise. Then, the subject performed ex- tension of the knee and dorsiflexion of the ankles simultaneously performed while flexion the upper cervical spine. This movement was performed until the patient felt pulling of the nerve tissue in the back of the thigh. After this, the subject performed upper cervical extension simultaneously with knee flexion and ankle plantar flexion. The subjects performed five sets of this exercise consecutively with active movement for approximately 30 seconds, taking a rest for 10 seconds in between sets. ${ }^{29}$ After resting for 2 minutes, they performed the same exercise using the opposite lower limb (Figure 3).

\section{Statistical analysis}

A statistical analysis of the data collected during measurements performed in this study was conducted using SPSS (v.18.0 for Windows; SPSS Inc., Chicago, IL, USA). The Kolmogorov-Smirnov test was performed to test normality distribution, and after the normality distribution was verified, a parameter test was conducted. An independent $t$ test was performed to verify the homogeneity of the slider and tensioner groups. The paired $t$-test was applied to com-

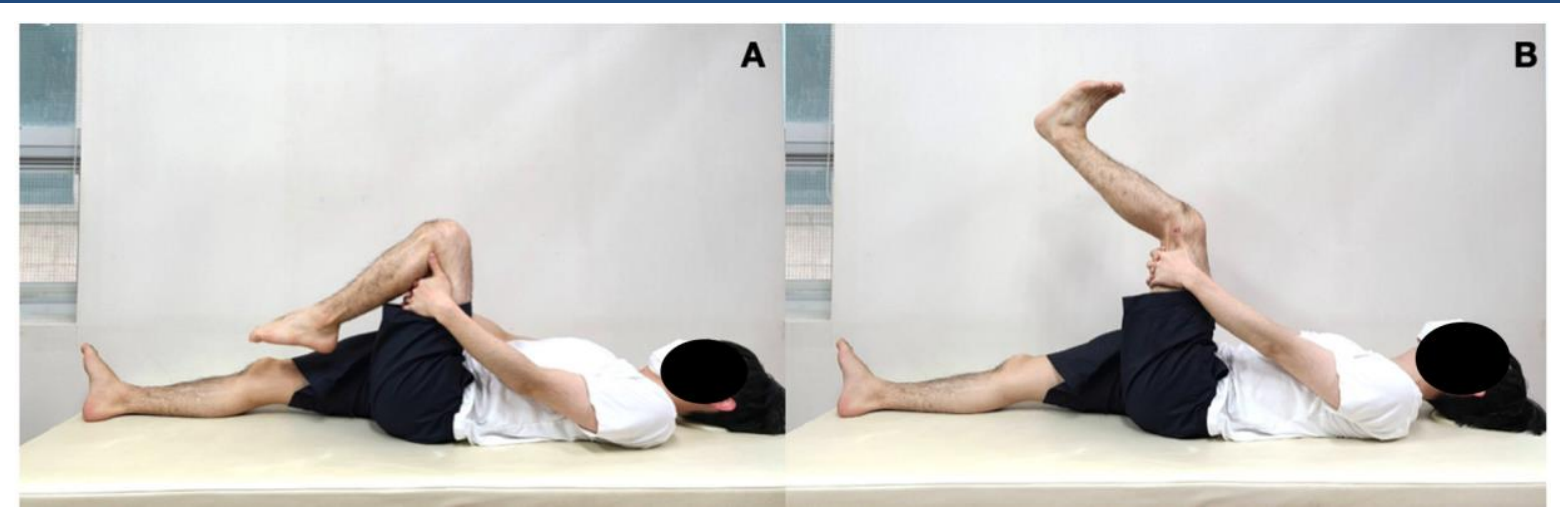

Figure 2. Slider (A: start position - upper cervical flexion, knee flexion, and ankle plantarflexion , B: end position - upper cervical extension, knee extension, and ankle dorsiflexion).

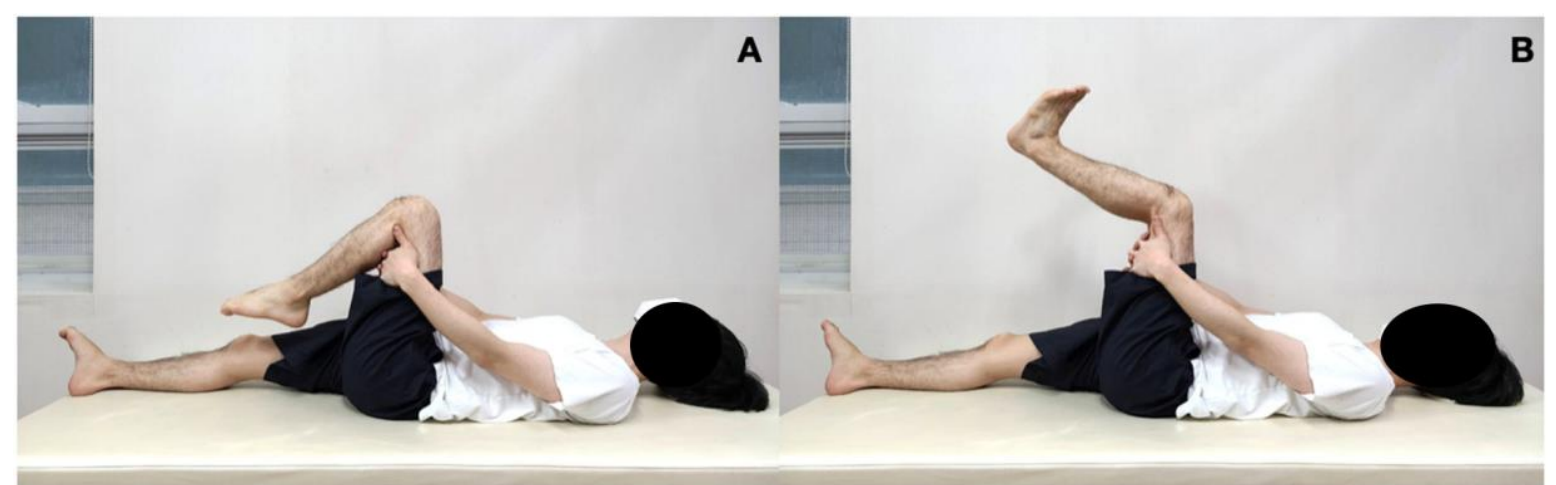

Figure 3. Tensioner (A: start position - upper cervical extension, knee flexion, and ankle plantarflexion, B: end position upper cervical flexion, knee extension, and ankle dorsiflexion). 
pare pre- and postintervention within the groups. The independent $t$-test was used to compare the change rates of hip and lumbar flexion ROMs (slider and tensioner). The statistical significance level was set at $p<.05$.

\section{RESULTS}

There were no significant differences in characteristics between the groups at the beginning of the study (Table 1). Intragroup comparison found that the slider group had a significant increase in hip flexion ROM at post-intervention (Table 2), whereas Intergroup comparison found a significant greater change in hip flexion ROM between pre- and postintervention in slider group (Table 3).

\section{DISCUSSION}

This study was to compare the effectiveness of forward bending using sliders versus tensioners in subjects with hamstring tightness. In particular, the study measured ROM using the Smart KEMA motion sensor. Although the ROM can be measured using a goniometer, it has a low reliability of measurement because the reference setting for the measurement procedure can be ambiguous. ${ }^{30}$ Hence, this study used the Smart KEMA software to improve the reliability of measurements. The smart KEMA motion sensor showed a very high reliability of 0.89 in the intraclass correlation coefficient value, which describes the test-retest reliability for hamstring flexibility. ${ }^{31}$

The intragroup comparison found that the hip flexion ROM of the slider group increased significantly during for- ward bending at post-intervention, but the lumbar flexion ROM did not show a significant difference. Furthermore, the intergroup comparison found a significant greater change in hip flexion ROM in slider group. The findings of the present study demonstrated that in subjects with hamstring tightness, sliders are more effective than tensioners in improving hip flexion ROM during forward bending. Neural mechanosensitivity is a normal protective mechanism that allows nerves to respond to mechanical stress during movement. ${ }^{28}$ The neural mechanosensitivity of the posterior leg, thigh, and buttock may play an important role in hamstring extensibility. The increased hip flexion ROM during forward bending means that the slider technique decreased the neural mechanosensitivity for hamstring flexibility. ${ }^{15}$ Although the use of sliders increased hamstring flexibility during forward bending, it is considered that the flexibility of lumbar extensors was not affected.

This finding is supported by previous studies. ${ }^{11,23}$ In a study of Castellote-Caballero et al., the effect of 1-week slider on asymptomatic male soccer players was compared between intervention and non-intervention groups. ${ }^{11}$ They performed a passive SLR to measure the change in the flexibility of the hamstring after intervention. ${ }^{11}$ As a result, it was reported that the intervention group was about 8.3 degrees higher than the non-intervention group. ${ }^{11}$ Park et al. investigated the immediate effect of the slider applied in the slump position on hamstring flexibility in asymptomatic subjects. ${ }^{23}$ The authors reported that the passive SLR immediately improved about 12 degrees after executing the slider in the slum position. ${ }^{23}$ The findings of these studies are consistency with the results of this study that the slider

Table 2. Comparison of the range of motion during forward bending in pre- and posttest within the group (unit: degree)

\begin{tabular}{cccccc}
\hline Group & & Pretest & Posttest & $t$ & $p$ \\
\hline \multirow{2}{*}{ Slider } & Hip flexion & $31.1 \pm 3.92$ & $40.87 \pm 4.26$ & -16.43 & $0.00^{*}$ \\
& Lumbar flexion & $37.28 \pm 3.12$ & $36.26 \pm 2.70$ & 1.17 & 0.27 \\
\multirow{2}{*}{ Tensioner } & Hip flexion & $30.62 \pm 7.64$ & $30.00 \pm 8.61$ & 0.72 & 0.48 \\
& Lumbar flexion & $39.73 \pm 4.15$ & $36.6 \pm 5.86$ & 1.60 & 0.14 \\
\hline
\end{tabular}

Data are expressed as mean \pm standard deviation; ${ }^{*} p<0.05$.

Table 3. Intergroup comparison of the changes in hip and lumbar flexion during forward bending (unit: degree)

\begin{tabular}{ccccc}
\hline Group & Slider & Tensioner & $t$ & $p$ \\
\hline Hip flexion (post-pre) & $9.77 \pm 1.88$ & $-0.62 \pm 2.69$ & 10.00 & $0.00^{*}$ \\
Lumbar flexion (post-pre) & $-1.02 \pm 2.74$ & $-3.13 \pm 6.17$ & 0.987 & 0.33 \\
\hline
\end{tabular}

Data are expressed as mean \pm standard deviation; ${ }^{*} p<0.05$. 
increased hamstring flexibility. But, there is a difference in that these studies used passive SLR tests to examine the effects of sliders, whereas the present study selected forward bending. The findings of current study may provide additional evidence for the effect of the neurodynamic slider on forward bending.

However, the intragroup comparison of the tensioner group did not show significant improvements in both hip and lumbar flexion ROMs during forward bending. Neto et al. measured the stiffness of the sciatic nerve while applying the tensioner technique to healthy adults and reported that tensioners did not decrease the stiffness of the sciatic nerve. ${ }^{32}$ In a similar study, Andrade et al. reported that the stiffness of the sciatic nerve increased during dorsiflexion of the ankles in an extended knee position when the tensioner was applied. ${ }^{33}$ The findings of these studies suggest that the tensioner could increase neural mechanosensitivity, and the protective contraction of the hamstring muscle increased. Such protective contraction is thought to be the cause for not changing hip and lumbar flexion ROMs during forward bending. In contrast to the findings of the present study, hamstring flexibility increased after the tensioner was applied in some studies. Fidel et al. applied tensioner mobilization in the slum position on 27 healthy subjects. They reported an increase of about 5.6 degrees in the range of AKE after tensioner mobilization. ${ }^{21}$ Furthermore, Sharma et al. investigated the additional effect of slider and tensioner on hamstring flexibility compared to static hamstring stretching. ${ }^{17}$ They reported that both techniques were more effective for hamstring flexibility than static stretching, but there was no significant difference in the effectiveness of the two techniques. ${ }^{17}$ However, their studies applied the tensioner technique in the slumped position and measured hamstring flexibility using knee extension ROM. The present study applied the tensioner technique in the supine position and measured hamstring flexibility using forward bending. Therefore, their studies and the present one could not be compared directly because of the differences in the tensioner application method and the measurement method. The differences in the tensioner application method and the hamstring flexibility measurement method should be improved further in the future.

The present study has a few limitations: First, the results of this study should not be interpreted as long-term effects because it compared immediate effects of the intervention methods. The duration of the effects could not be determined. Second, all the subjects were young men. Therefore, the results of this study may differ from those performed on women or other age groups. Third, the neurodynamic technique used in this study was carried out in the supine position. Therefore, it should not be interpreted as a result of the neurodynamic technique performed in another position. In the future, the long-term effects of various interventions that supplement these limitations need to be explored.

\section{CONCLUSIONS}

This study demonstrated that the slider technique significantly improved hip flexion ROM during forward bending in subjects with hamstring tightness compared to the tensioner technique. These data suggest that sliders may be useful in increasing hamstring flexibility during forward bending.

\section{Key Points}

Question What type of neurodynamic technique is more effective in increasing hamstring flexibility during forward bending in subjects with hamstring tightness?

Findings Intra- and inter-group comparisons found that the hip flexion angle during forward bending after using the slider increased more significantly compared to the tensioner. However, there was no significant difference in the lumbar flexion angle.

Meaning This suggests that the slider is more effective in increasing hamstring flexibility than the tensioner on forward bending in subjects with hamstring tightness.

\section{Article information}

Conflict of Interest Disclosures: None.

Funding/Support: None.

Acknowledgment: None.

Ethic Approval: The study was approved by the Ethics and Research Committee of Daegu Health College (\#DHCIRB-2020-09-0006).

\section{REFERENCES}

1. Youdas JW, Krause DA, Hollman JH, Harmsen WS, Laskowski E. The influence of gender and age on hamstring muscle length in healthy adults. J Ortho Sports Phys Ther. 2005;35(4): 246-252.

2. Norris C, Matthews M. Correlation between hamstring muscle length and pelvic tilt range during forward bending in healthy individuals: An initial evaluation. $J$ Bodyw Mov Ther. 2006; 10(2):122-126.

3. Esola MA, McClure PW, Fitzgerald GK, Siegler S. Analysis of lumbar spine and hip motion during forward bending in subjects with and without a history of low back pain. Spine. 1996;21(1):71-78. 
4. Jones M, Stratton G, Reilly T, Unnithan V. Biological risk indicators for recurrent non-specific low back pain in adolescents. Br J Sports Med. 2005; 39(3):137-140.

5. Ayala F, Sainz de Baranda P, De Ste Croix M, Santonja F. Comparison of active stretching technique in males with normal and limited hamstring flexibility. Phys Ther Sport. 2013;14(2):98-104.

6. Carregaro RL, Coury HJCG. Does reduced hamstring flexibility affect trunk and pelvic movement strategies during manual handling? Int J Ind Ergon. 2009;39(1): 115-120.

7. Sahrmann S. Diagnosis and treatment of movement impairment syndromes. St Louis: Mosby; 2002.

8. Nourbakhsh MR, Arab AM. Relationship between mechanical factors and incidence of low back pain. $J$ Ortho Sports Phys Ther. 2002;32(9):447-460.

9. Hasebe K, Okubo Y, Kaneoka K, Takada K, Suzuki D, Sairyo K. The effect of dynamic stretching on hamstrings flexibility with respect to the spino-pelvic rhythm. J Med Invest. 2016;63(1.2):85-90.

10. Li Y, McClure PW, Pratt N. The effect of hamstring muscle stretching on standing posture and on lumbar and hip motions during forward bending. Phys Ther. 1996;76(8):836-845.

11. Castellote-Caballero Y, Valenza MC, Martín-Martín L, Cabrera-Martos I, Puentedura EJ, Fernández-de-lasPeñas C. Effects of a neurodynamic sliding technique on hamstring flexibility in healthy male soccer players. A pilot study. Phys Ther Sport. 2013;14(3): 156-162.

12. Choi K-h. Comparison of the anterior pelvic tilting angle during forward bending in individuals with and without sciatic nerve tension. J Musculoskelet Sci Technol. 2019;3(2):44-48.

13. Méndez-Sánchez R, Alburquerque-Sendín F, Fernándezde-las-Peñas C, et al. Immediate effects of adding a sciatic nerve slider technique on lumbar and lower quadrant mobility in soccer players: A pilot study. $J$ Altern Complement Med. 2010;16(6):669-675.

14. Shacklock M. Clinical neurodynamics: A new system of neuromusculoskeletal treatment. London: Elsevier Health Sciences; 2005.

15. Castellote-Caballero Y, Valenza MC, Puentedura EJ, Fernández-de-las-Peñas C, Alburquerque-Sendín F. Immediate effects of neurodynamic sliding versus muscle stretching on hamstring flexibility in subjects with short hamstring syndrome. J Sports Med. 2014; 2014.

16. Pagare VK, Ganacharya PM, Sareen A, Palekar TJ. Effect of neurodynamic sliding technique versus static stretching on hamstring flexibility in football players with short hamstring syndrome. J Musculoskelet Res. 2014;
17(02): 1450009.

17. Sharma S, Balthillaya G, Rao R, Mani R. Short term effectiveness of neural sliders and neural tensioners as an adjunct to static stretching of hamstrings on knee extension angle in healthy individuals: A randomized controlled trial. Phys Ther Sport. 2016;17:30-37.

18. Butler DS. Mobilization of the nervous system. J Hand Ther. 1994;7:33-33.

19. Singh S, Grover V, Singh S. Effect of neural mobilization and pnf stretching on hamstring flexibility in working women. Int J Health Sci Res. 2015;5(8):361368.

20. Coppieters MW, Hough AD, Dilley A. Different nervegliding exercises induce different magnitudes of median nerve longitudinal excursion: An in vivo study using dynamic ultrasound imaging. J Ortho Sports Phys Ther. 2009;39(3):164-171

21. Fidel C, Martin E, Dankaerts W, Allison G, Hall T. Cervical spine sensitizing maneuvers during the slump test. J Man Manip Ther. 1996; 4(1):16-21.

22. Shacklock M. Clinical neurodynamics: a new system of musculoskeletal treatment. London: Elsevier Health Sciences; 2005.

23. Park J-m, Cha J-Y, Kim H-J, Yasuyoshi A. Immediate effects of a neurodynamic sciatic nerve sliding technique on hamstring flexibility and postural balance in healthy adults. Phys Ther Rehabil Sci. 2014;3(1):38-42.

24. Bandy WD, Irion JM, Briggler M. The effect of time and frequency of static stretching on flexibility of the hamstring muscles. Phys Ther. 1997;77(10):1090-1096.

25. Hamid MSA, Ali MRM, Yusof A. Interrater and intrarater reliability of the active knee extension (AKE) test among healthy adults. J Phys Ther Sci. 2013;25(8): 957-961.

26. Reis FJJ, Macedo AR. Influence of hamstring tightness in pelvic, lumbar and trunk range of motion in low back pain and asymptomatic volunteers during forward bending. Asian Spine J. 2015;9(4):535.

27. Gamelas T, Fernandes A, Magalhães I, Ferreira M, Machado S, Silva AG. Neural gliding versus neural tensioning: Effects on heat and cold thresholds, pain thresholds and hand grip strength in asymptomatic individuals. J Bodyw Mov Ther. 2019;23(4):799-804.

28. Nee RJ, Butler D. Management of peripheral neuropathic pain: Integrating neurobiology, neurodynamics, and clinical evidence. Phys Ther Sport. 2006; 7(1):3649.

29. Singh AK, Nagaraj S, Palikhe RM, Neupane B. Neurodynamic sliding versus PNF stretching on hamstring flexibility in collegiate students: A comparative study. 
Int J Sports Health Phys Educ. 2017;1(1):29-33.

30. Kilgour G, McNair P, Stott NS. Intrarater reliability of lower limb sagittal range-of-motion measures in children with spastic diplegia. Dev Med Child Neurol. 2003; 45(6):391-399.

31. Kim J-s, Hwang U-j, Kwon O-y, Park K-n. Reliability of hamstring flexibility test with hip inferior glide mobilization in patients with low back pain. J Musculoskelet Sci Technol. 2019;3(1): 26-30.
32. Neto T, Freitas SR, Andrade RJ, et al. Sciatic nerve stiffness is not changed immediately after a slump neurodynamics technique. Muscles Ligaments Tendons J. 2017;7(3):583.

33. Andrade RJ, Nordez A, Hug F, et al. Non-invasive assessment of sciatic nerve stiffness during human ankle motion using ultrasound shear wave elastography. $J$ Biomech. 2016;49(3):326-331. 